\title{
A cultural study on decision making: performance differences on the Iowa gambling task between selected groups of brazilians and americans
}

\author{
Daniela Schneider Bakos ${ }^{1}$, Natalie Denburg ${ }^{2}$, Rochele Paz Fonseca ${ }^{3}$ and Maria Alice de Mattos \\ Pimenta Parente ${ }^{4}$ \\ 1 Universidade Luterana do Brasil, Brazil \\ 2 University of Iowa, USA \\ 3 Pontifícia Universidade Católica do Rio Grande do Sul, Brazil \\ 4 Universidade Federal do Rio Grande do Sul, Brazil
}

\begin{abstract}
This study compared the performance of selected groups of Brazilian and American individuals on the Iowa Gambling Task (IGT). The sample was composed of 70 Brazilian and American individuals of both genders, divided into two groups of young and older adults. The two groups of Brazilians were composed of 10 young adults, aged 25 to 41 years, and 25 older adults, aged 60 to 75 years. The two groups of Americans were composed of 10 young adults, aged 28 to 39 years, and 25 older adults, aged 60 to 76 years. A two-way analysis of variance revealed differences in performance between Brazilian and American participants, regardless of age group (country, $p=.001$; age group, $p=.134$; country $v s$. age group, $p=.291$ ). Analyses of variance using age group and country as main factors did not reveal any difference between the two national groups with regard to the learning achieved during the task (block, $p<.001$; age group, $p=.063$; country, $p=.086$ ). The results suggest that culture, represented by the country of origin, influences the performance of individuals on the IGT, demonstrating the importance of validating an instrument for each cultural group on which it is used. Keywords: decision making, Iowa Gambling Task, culture.
\end{abstract}

Received 21 January 2010; received in revised form 29 March 2010; accepted 5 Abril 2010. Available on line 26 June 2010

\section{Introduction}

Although the impact of cultural factors on subjects' performance on neuropsychological tests was demonstrated by Luria and Vygotsky in the 1930s, only in the past few decades has an increasing number of publications investigated normative data, which allow for the speedier development of neuropsychological instruments adapted or validated for specific populations (Agronovich \& Puente, 2007; Ardila, 1995; Brickman,

Daniela Schneider Bakos, Department of Psychology, Universidade Luterana do Brasil, Porto Alegre, Rio Grande do Sul, Brazil. Natalie Denburg, Department of Neurology, University of Iowa, Iowa City, Iowa, United States of America. Rochele Paz Fonseca, Department of Psychology, Pontifícia Universidade Católica do Rio Grande do Sul, Porto Alegre, Rio Grande do Sul, Brazil. Maria Alice de Mattos Pimenta Parente, Department of Psychology, Universidade Federal do Rio Grande do Sul, Porto Alegre, Rio Grande do Sul, Brazil. Correspondence regarding this article should be directed to: Daniela Schneider Bakos, Rua Arthur Rocha, 669 / 704, Porto Alegre, RS, CEP 90450-171, Brazil. E-mail: daniela@clinicacognita.com.br
Cabo, \& Manly, 2006; Hsieh \& Tori, 2007; Manly, 2008). As the field of clinical neuropsychological continues to evolve, a number of assumptions related to cognitive neuropsychology, experimental psychology, psycholinguistics, and psychometric properties is becoming explicitly examined (Fonseca, Parente, Côté, \& Joanette, 2007; Hsieh \& Tori, 2007; Manly, 2008).

Cross-cultural factors affecting neuropsychological assessments have been analyzed in many studies involving distinct cognitive functions. This growing interest is reflected by cross-cultural investigations of attention (Rosselli, Tappen, Williams, \& Salvatierra, 2006), perception (Herrera-Guzman, Pena-Casanova, Lara, Gudayol-Ferre, \& Bohm, 2004), memory (Johnson, Storandt, \& Balota, 2003), language (Lecours et al., 1987; Radanovic, Mansur, \& Scaff, 2004), and executive function (Ostrosky-Solis, Gutierrez, Flores, \& Ardila, 2007; Plumet, Gil, \& Gaonac'h, 2005).

In the context of neuropsychological assessment of executive function, the Iowa Gambling Task (IGT) (Bechara, Damasio, Damasio, \& Anderson, 1994) has been widely used to investigate decision making (DM). Despite 
the widespread use of the IGT in different cultural groups (Bechara, 2003; Bechara et al., 2001; García-Molina, Rodríguez, Vendrell Gómez, Junqué, \& Roig-Rovira, 2008; Tranel, Bechara, \& Denburg, 2002; Verdejo-García, Bechara, Recknor, \& Pérez-García, 2007), the cultural effects on IGT scores have not been directly addressed. Some investigations have indicated confounding factors affecting the performance of different cultural groups on the task, but still unclear is whether these differences are related to cultural variables. For example, one study found that normal Taiwanese subjects prefer cards from deck " $\mathrm{B}$ " and dislike cards from deck "C" of the IGT (Chiu \& Lin, 2007; Lin, Chiu, Lee, \& Hsieh, 2007). Therefore, more research needs to be conducted to investigate the influence of cultural and sociodemographic factors on individuals' performance on this task.

The role of gender in IGT scores is also somewhat ambiguous. Although some studies have reported an absence of such an influence in young and adult samples (Davis, Patte, Tweed, \& Curtis, 2007; Franken \& Muris, 2005), other investigations have found that men and women exhibited differential performance (Bolla, Eldreth, Matochik, \& Cadet, 2004; Goudriaan, Grekin, \& Sher, 2007; Reavis \& Overman, 2001). The study conducted by Goudriaan et al. (2007) in a population of young people with the habit of consuming alcoholic beverages indicated that women more often chose decks with low frequency but very costly punishments.

With regard to the influence of education on individuals' performance on the IGT, worse performance has been observed with higher education levels (Evans, Kemish, \& Turnbull, 2004), although further evidence of this effect of education is still needed. Furthermore, normative data from a sample of 932 healthy participants minimized the importance of educational level by showing that years of schooling accounted for only $0.3 \%$ of a $3.6 \%$ variance between IGT scores (Bechara, 2007).

The influence of age on DM, measured by the IGT, is supported by numerous studies, although some results have been controversial. Some studies demonstrated no difference between young and older adults with regard to the number of cards chosen from each deck (Lamar \& Resnick, 2004; MacPherson, Phillips, \& Sala, 2002; Schneider \& Parente, 2006; Wood, Busemeyer, Koling, Cox, \& Davis, 2005), but other studies found that elderly individuals as a group performed worse than younger adults (Deakin, Aitken, Robbins, \& Sahakian, 2004; Denburg, Bechara, Cole, \& Tranel 2001; Denburg, Recknor, Bechara, \& Tranel, 2006; Denburg, Tranel, \& Bechara, 2005; Fein, McGillivray, \& Finn, 2007).

Considering the possible role of age in the DM process, an interaction between this variable and culture can be expected, such that culture may differentially influence individuals in different age groups. For example, an investigation performed by Baird, Ford and Podell (2007) demonstrated that elderly American individuals of European descent performed better on naming tasks, visual patterns, and skills related to money than elderly American individuals of African descent. Although this study found evidence of a cultural effect on the performance of specific cognitive tasks by elderly individuals, it did not establish an association between performance and other age groups.

The present study assessed performance differences on the IGT between selected groups of Brazilians and Americans. Iowa Gambling Task performance was compared between selected groups of American and Brazilian young and elderly adults.

\section{Methods}

\section{Participants}

The sample was composed of 70 Brazilian and American individuals of both genders, divided into two groups of young and elderly individuals. The Brazilian cohort contained 10 young adults, aged 25 to 41 years, and 25 elderly individuals, aged 60 to 75 years. The American groups were similarly distributed: 10 young adults, aged 28 and 39 years, and 25 elderly individuals, aged 60 to 76 years. The Brazilian participants were selected from universities and social centers catering to the elderly, whereas most of the American data had previously been recorded at the University of Iowa Hospitals and Clinics' Department of Neurology. American participants were paid for their participation, whereas the Brazilian participants were not. Inclusion criteria were the absence of psychological disorders, dementia, uncorrected sight, and hearing disorders and familiarity with the tests or frequent involvement in financial investments. The minimum level of education of the participants enrolled in this study was $\geq 8$ years, which is the equivalent of an elementary school (i.e., primary school) education. To eliminate the possible influence of other sociodemographic variables, such as gender, educational level, and age, the groups were carefully matched. The samples were also matched for their cognitive status, based on their scores on the digit span and vocabulary subtests and the Mini-Mental State Examination (MMSE). See Table 1 for the sociodemographic characteristics of the participants.

\section{Instruments and procedures}

The same instruments were administered to both national groups. These included the digit span and vocabulary subtests (Wechsler Adult Intelligence Scale, WAIS-III) (Wechsler, 1997) and the IGT (Bechara et al., 1994). The instruments used in Brazil were originally American, and the Brazilian versions of the subtests of the WAIS-III were previously validated (Nascimento, 2000). The following instruments were administered to each Brazilian participant individually in a single session at the Neuropsycholinguistics Laboratory of 
Table 1. Sociodemographic characteristics of the sample.

\begin{tabular}{lcccc}
\hline & \multicolumn{2}{c}{ Group } & \multicolumn{1}{c}{ American Adults } \\
\cline { 2 - 5 } & $\begin{array}{c}\text { Young } \\
\text { (n=10) }\end{array}$ & $\begin{array}{c}\text { Elderly } \\
(n=25)\end{array}$ & $\begin{array}{c}\text { Young } \\
(n=10)\end{array}$ & $\begin{array}{c}\text { Elderly } \\
(n=25)\end{array}$ \\
\hline Gender (male/female) & $(3 / 7)$ & $(6 / 19)$ & $(3 / 7)$ & $(6 / 19)$ \\
Age (years; mean/SD) & $32.35 / 3.72$ & $68.16 / 5.40$ & $32.35 / 3.72$ & $68.16 / 5.40$ \\
Level of education (years; mean/SD) & $16.05 / 2.42$ & $13.98 / 2.97$ & $16.05 / 2.42$ & $13.98 / 2.97$ \\
MMSE (mean/SD) & - & $29.10 / 1.42$ & - & $29.10 / 1.42$ \\
Digit Span Subtest score & $13.00 / 2.66$ & $11.84 / 2.44$ & $12.20 / 3.32$ & $10.84 / 2.91$ \\
Vocabulary Subtest score & $11.50 / 1.65$ & $11.24 / 1.69$ & $12.00 / 2.26$ & $11.92 / 2.27$ \\
\hline
\end{tabular}

The MMSE was administered only to the elderly groups. SD, standard deviation.

the Universidade Federal do Rio Grande do Sul or at the participant's home. Before administering the IGT, the examiner administered the following screening instruments: MMSE (Folstein, Folstein, \& McHugh, 1975; adapted for Brazilian Portuguese by Chaves \& Izquierdo, 1992), the Modified Mini International Neuropsychiatry Interview (MINI) (Sheehan et al., 1998; validated for the Brazilian population by Amorim, 2000), and a Demographic Data Questionnaire. The MMSE was only used with elderly individuals because most of the young adults were college students who were regularly engaged in intellectual activity. All screening instruments were administered manually, whereas the IGT was completed in its computerized version. Participation in this study was voluntary, and all participants signed a consent form. The project was approved by the Research Ethics Committee of the Institute of Psychology of the Universidade Federal do Rio Grande do Sul (no. 2006/005).

The tests were individually administered to the American participants in a single session at the University of Iowa Hospitals and Clinics' Department of Neurology. The participants were first contacted by telephone so they could be given information regarding the study's objectives and their participation. Following the participant's agreement to participate in the study, a meeting was scheduled according to his or her availability. The screening instruments were administered first, followed by the IGT. All screening instruments were presented manually, and the IGT was administered in its computerized version. Participation in this study was voluntary, and all participants signed a consent form. The individuals received the equivalent of USD $\$ 12.00$ per hour for their participation in the study. All research assistants attended an online course called, "Human Participant Protections Education for Research Teams," supported by the U.S. National Institutes of Health.
Iowa Gambling Task

A computerized version of the IGT, developed by Bechara et al. (1994) and adapted to Brazilian Portuguese by Schneider and Parente (2006), was used to assess DM in the Brazilian sample. The American group was given the original task developed by Bechara et al. (1994). Both versions of the IGT are administered identically. This instrument involves DM under conditions of uncertainty and requires participants to make short- and long-term monetary decisions. The IGT allows for classification of an individual's DM behavior in terms of adaptive or impaired DM skills.

The task consists of choosing cards from four decks for a total of 100 choices (five groups of 20 choices each). Each deck has a fixed and preprogrammed system of rewards and punishments, based on a learning process, and participants can create likelihood patterns and infer which decks are advantageous and which are not by considering both short-term and long-term risks and benefits.

\section{Data analysis}

Participants' scores on the IGT were compared between the two national groups. The results were based on two dependent variables. The first refers to the total net score achieved in the task, calculated by subtracting the number of cards chosen from disadvantageous decks from those chosen from advantageous decks: $(C+D)$ $-(A+B)$. This score is referred to as the calculationbased score (mental) in the present study. The second variable is the net score obtained in each of the five blocks of 20 cards throughout the 100 choices. The formula used to obtain this result is calculated every 20 cards and is the same as the one used to calculate the calculation-based score (mental): $(C+D)-(A+$ $B)$. Verifying whether participants showed evidence of learning while performing the task is possible by using these scores (Bechara, 2007). 


\section{Results}

To investigate whether the two national groups differed in the final score obtained on the task, based on the formula $(A+B)-(C+D)$, a two-way analysis of variance (ANOVA) was performed, with age group and country as the main factors. The results showed that the performance of Brazilian and American participants differed, regardless of age group. More specifically, American participants, as a group, performed better on the task (country, $p \leq .001$; age group, $p=.134$; country vs. age group, $p=.291$ ) than Brazilian participants.

Repeated-measures ANOVAs, with age group and country as main factors, did not reveal any differences between the two national groups in the learning achieved throughout the task. Learning is defined as the scores obtained on the five successive blocks of 20 cards each. The results showed that some learning occurred during the task, but it did not differ as a function of age group or country (block, $p \leq .001$; age group, $p=.063$; country, $p=.086)$ (Figure 1).

To analyze individual performance in the two groups, a single score was calculated for each participant using the formula $(C+D)-(A+B)$ to obtain the final result in terms of choices made throughout the task. After this result was determined, cut-off points were established based on a binomial distribution (Denburg et al., 2005) to classify individual performance as impaired (score $<18$ ), borderline $(-18<$ score $<+18)$, or non-impaired (score $\geq 18$ ). The $\chi^{2}$ test indicated a significant difference between groups $(p \leq .001)$. The American sample had significantly more non-impaired participants than the Brazilian sample. Such differences were also found

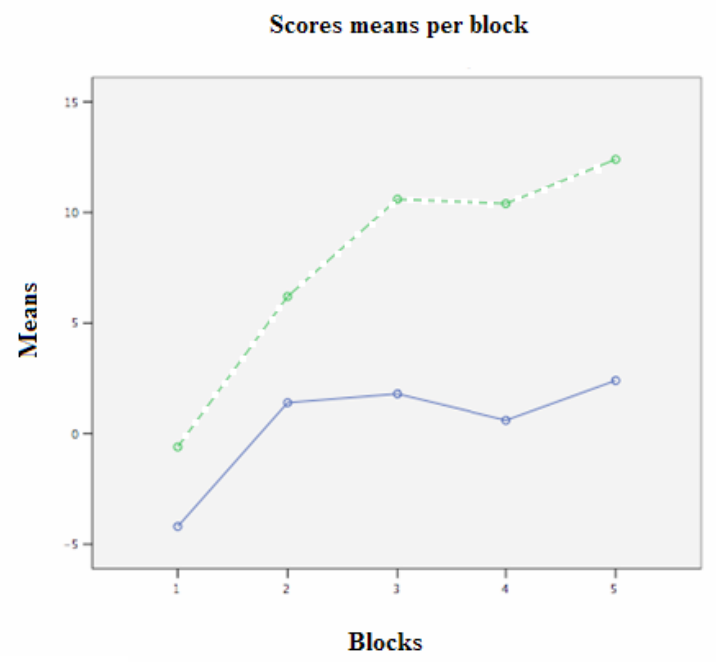

$$
\begin{aligned}
& \text { Countries } \\
& \text { - Brazil } \\
& \text { - USA }
\end{aligned}
$$

Figure 1. Mean score of the groups per block. when the analysis was performed according to age group alone. The performance of non-impaired participants in the sample is shown in Table 2.

Table 2. Iowa Gambling Task scores of non-impaired Brazilian and American subjects.

\begin{tabular}{lcc}
\hline & \multicolumn{2}{c}{ Group } \\
\cline { 2 - 3 } & Brazilians & Americans \\
\hline Non-impaired & $17 \%$ & $60 \%$ \\
Impaired & $83 \%$ & $40 \%$ \\
Borderline & $0 \%$ & $0 \%$ \\
\hline
\end{tabular}

\section{Discussion}

The results of the present study revealed that total IGT scores were significantly greater in American individuals compared with Brazilian participants, indicating that the former demonstrated higher DM skills on this task. More specifically, our findings demonstrated that, when choosing financial options, more than half of the Americans in our sample chose alternatives that would be advantageous in the long-term, whereas about $80 \%$ of the Brazilian participants made disadvantageous choices. Nevertheless, the learning achieved during the task did not differ between the two groups, suggesting that they had similar choice-based learning skills but different DM strategies. This discussion focuses on the differences in total scores and the absence of different learning processes.

Some authors have discussed the role of sociodemographic characteristics, such as gender (Goudriaan et al., 2007; Reavis \& Overman, 2001) and years of schooling (Evans et al., 2004), in IGT performance. In the present study, these variables were properly controlled and, therefore, cannot explain the results. Similarly, age, which has been reported in other studies to play a major role in individuals' performance on the IGT (Deakin et al., 2004; Denburg et al., 2001, 2005, 2006; Fein et al., 2007), especially as a result of inherent biological changes, had no influence on our sample's performance.

The cognitive status of the present sample, with regard to the other cognitive processes involved in DM measured by the IGT, may also be a contributing factor to the observed findings. We attempted to mitigate the impact of cognitive status on DM by pairing the groups as a function of their scores on the MMSE and digit span and vocabulary subtests. Nevertheless, the influence of cognitive status on the results was evaluated, and no significant effect was found, although one must consider this a limitation given that the tests cannot not be assumed to measure the same construct or skill in Brazilian, Portuguese-speaking subjects compared with American, English-speaking subjects. 
Consequently, alternative explanations should be sought for the better performance exhibited by the American individuals. One possibility is the effect of culture on the participants' behavior. A series of ongoing studies have shown that the cognitive processes required for completing neuropsychological tests are dependent on cultural experience. Some aspects of culture, including the relevance of certain cognitive abilities for daily activities, may affect how well individuals acquire the skills needed to perform these tests, even among healthy samples (Razani, Burciaga, Madore, \& Wong, 2007). More specifically, the demands of each culture, reflected in the daily activities of a given society, result in different ways of conceptually processing information and may increase the acquisition of specific cognitive skills and processing strategies to the detriment of others.

Based on this hypothesis, Ardila (1995) claimed that cognitive skills, beliefs, and behaviors are culturally learned; therefore, they are cultural elements. This author stated that individuals' scores on neuropsychological tests correlate with their learning opportunities and contextual experience, which define what is relevant to be learned and at what age.

In the present sample, culture can be hypothesized to be a factor that influenced the difference in performance between the two groups. Americans, as a group, can be posited to have better DM skills because of more daily demands on related cognitive processes, especially those that involve economic issues. Some authors have suggested in cross-cultural studies that better scores are usually obtained by the population in which the instrument was developed because it reflects the test developer's culture and own way of perceiving the world (Razani et al., 2007).

Another important issue regarding cultural differences concerns how individuals conceptualize money, risk, losses, and gains. These factors should be considered because they may be different in different countries and may influence the participants' attitudes during the task. This may occur because the way people conceptualize these variables may be associated with how they act toward them. A markedly capitalistic culture, such as the American culture, may bias an individual toward an increased focus on financial issues. Moreover, they may be more familiar with financial calculations and decisions than an individual from a less capitalist-oriented culture.

The participant's attitude toward the testing situation has also been suggested to be a factor that may differ among cultures and may influence a subject's performance during the assessment (Manly \& Echemendia, 2007; Manly et al., 1998). Manly and colleagues (1998) reported the importance of "stereotype threat," a concept that refers to an individual's concern that poor performance will confirm a previously existing negative social stereotype. Brazilian participants who are willing to be tested usually come from lower social classes and sometimes reveal a fear of making mistakes. Elderly participants must also face certain social clichés concerning their limitations and deficits. Such attitudes have been suggested to be less common in American society because more developed countries have a better distribution of resources and more adequate support for the elderly.

Additionally, particular features of the testing situation, such as administration time and financial incentives, may also play a relevant role. Such factors act on motivational components that have been associated with IGT performance (Yechiam, Busemeyer, Stout, \& Bechara, 2005) and may contribute to losses as a result of fatigue or lack of motivation. Despite a study showing no difference between real $v s$. facsimile reinforcers on the IGT, the financial incentive was not directly addressed in the present investigation. Therefore, one might also assume that the American participants were more motivated because they earned a financial incentive to participate in the study. Furthermore, many American participants were familiar with the testing situation, a fact that might facilitate engagement (Agranovich \& Puente, 2007; Razani et al., 2007).

Reference can also be made to educational experience, which, according to some authors, is more important than the traditional quantitative measure of years of schooling (Brickman et al., 2006; Manly et al., 1998). Although the influence of educational level on individuals' performance on the IGT is still quite controversial, considering teaching quality may be important, which can vary between countries as a consequence of different educational systems. Measurements based on the number of years of formal schooling would be insufficient in this case. We tried to minimize this limitation by measuring intelligence aspects through digit and vocabulary scores, which are frequently associated with reading level, an educational qualitative factor that has been considered to be an important predictor of premorbid verbal intelligence in different neurological impairments (Baird et al., 2007; Manly, Jacobs, Touradji, Small, \& Stern, 2002; Schmand, Smit, Geerlings, \& Lindeboom, 1997). The pairing of the sample based on their digit span and vocabulary subtest scores, although not equivalent to matching education experience, may mitigate the differences in educational experience.

The findings of the present study indicate that culture, represented by the country of origin, at least partially influences the behavior of individuals on the IGT. Differences in the performance of different cultural groups on neuropsychological tests highlight the importance of validating and standardizing an instrument for each particular cultural group rather than interpreting performance based on the norms of another culture. Considering that no other studies have investigated the role of culture in IGT performance, the 
present results should be considered preliminary. Such an investigation, although essentially exploratory, is extremely useful for clinical practice because it suggests possible alternatives to overcome this limitation.

Some methodological limitations require cautious consideration of the findings of the present study. One is the small sample size, which restricts the generalizability of the data. Additionally, measuring years of education does not appear to be sufficient to ensure that the groups are matched for education levels. Considering the quality and location of education will be necessary to determine the educational systems' similarities and differences. Moreover, the Brazilian subjects were selected in a very different way than the American participants because Brazilian research participants cannot be paid for their participation as volunteers. Furthermore, the locations of data collection were not controlled. In Brazil, data were collected at a laboratory or at the subject's home. In the United States, data were collected at a university department. This is an important variable that might also contribute to some of the differences found in this report. Finally, the groups were not matched for computer literacy, which could play a role in completing a computerized version of the IGT. Consequently, we can assume that the groups were not completely matched because of the procedural differences in each country. Thus, future studies should use larger samples and control for the aspects mentioned above to elucidate the numerous cultural components operating within cross-cultural research designs.

\section{References}

Agranovich, A.V., \& Puente, A.E. (2007). Do Russian and American normal adults perform similarly on neuropsychological tests? Preliminary findings on the relationship between culture and test performance. Archives of Clinical Neuropsychology, 22, 273-282.

Amorim, P. (2000). Mini international neuropsychiatric interview (M.I.N.I.): Validação de entrevista breve para diagnóstico de transtornos mentais. Revista Brasileira de Psiquiatria, 3, 106-115.

Ardila, A. (1995). Directions of research in cross-cultural neuropsychology. Journal of Clinical and Experimental Neuropsychology, 17, 143-150.

Baird, A.D., Ford, M., \& Podell, K. (2007). Ethnic differences in functional and neuropsychological test performance in older adults. Archives of Clinical Neuropsychology, 22, 309-318.

Bechara, A. (2003). Risky business: emotion, decision-making, and addiction. Journal of Gambling Studies, 19, 23-51.

Bechara, A. (2007). Iowa Gambling Task: Professional manual. Odessa, FL: Psychological Assessment Resources.

Bechara, A., Damasio, A.R., Damasio, H., \& Anderson, S.W. (1994). Insensitivity to future consequences following damage to human prefrontal cortex. Cognition, 50, 7-15.

Bechara, A., Dolan, S., Denburg, N., Hindes, A., Anderson, S.W., \& Nathan, P.E. (2001). Decision-making deficits, linked to a dysfunctional ventromedial prefrontal cortex, revealed in alcohol and stimulant abusers. Neuropsychologia, 39, 376-389.

Bolla, K.I., Eldreth, D.A., Matochik, J.A., \& Cadet, J.L. (2004). Sex-related differences in a gambling task and its neurological correlates. Cerebral Cortex, 14, 1226-1232.

Brickman, A.M., Cabo, R., \& Manly, J.J. (2006). Ethical issues in crosscultural neuropsychology. Applied Neuropsychology, 13, 91-100.

Chaves, M.L., \& Izquierdo, I. (1992). Differential diagnosis between dementia and depression: a study of efficiency increment. Acta
Neurologica Scandinavica, 85, 378-382.

Chiu, Y.C., \& Lin, C.H. (2007). Is deck C an advantageous deck in the Iowa Gambling Task? Behavioral and Brain Functions, 3, ArtID 37.

Davis, C., Patte, K., Tweed, S., \& Curtis, C. (2007). Personality traits associated with decision-making deficits. Personality and Individual Differences, 42, 279-290.

Deakin, J., Aitken, M., Robbins, T., \& Sahakian, B.J. (2004). Risk taking during decision-making in normal volunteers changes with age. Journal of the International Neuroscience Society, 10, 590-598.

Denburg, N.L., Bechara, A., Cole, C., \& Tranel, D. (2001). Iowa Gambling Task performance in elderly persons predicts susceptibility to the influence of misleading advertising. Society for Neuroscience Abstracts, 27, Abstract\# 80.13.

Denburg, N.L., Recknor, E.C., Bechara, A., \& Tranel, D. (2006). Psychophysiological anticipation of positive outcomes promotes advantageous decision-making in normal older persons. International Journal of Psychophysiology, 61, 19-25.

Denburg, N.L., Tranel, D., \& Bechara, A. (2005). The ability to decide advantageously declines prematurely in some normal older persons. Neuropsychologia, 43, 1099-1106.

Evans, C.E.Y., Kemish, K., \& Turnbull, O.H. (2004). Paradoxical effects of education on the Iowa Gambling Task. Brain and Cognition, 54, 240-244.

Fein, G., McGillivray, S., \& Finn, P. (2007). Older adults make less advantageous decisions than younger adults: cognitive and psychological correlates. Journal of the International Neuropsychology Society, 13, 480-489.

Folstein, M.F., Folstein, S.E., \& McHugh, P.R. (1975). "Mini-mental state": a practical method for grading the cognitive state of patients for the clinician. Journal of Psychiatry Resources, 12, 189-198.

Fonseca, R.P., Parente, M.A.M.P., Côté, H., \& Joanette, Y. (2007). Processo de adaptação da bateria Montreal de Avaliação da Comunicação - Bateria MAC - ao Português Brasileiro. Psicologia: Reflexão e Crítica, 20, 259-267.

Franken, I.H.A., \& Muris, P. (2005). Individual differences in decisionmaking. Personality and Individual Differences, 39, 991-998.

García-Molina, A., Rodríguez, R.P., Vendrell Gómez, P., Junqué, P.C., \& Roig-Rovira, T. (2008). .Disfunción orbitofrontal en la esclerosis múltiple: Iowa Gambling Task. Psicothema, 20, 445-449.

Goudriaan, A.E., Grekin, E.R., \& Sher, K.J. (2007). Decision making and binge drinking: a longitudinal study. Alcoholism: Clinical and Experimental Research, 31, 928-938.

Herrera-Guzman, I., Pena-Casanova, J., Lara, J.P., Gudayol-Ferre, E., \& Bohm, P. (2004). Influence of age, sex, and education on the Visual Object and Space Perception Battery (VOSP) in a healthy normal elderly population. Clinical Neuropsychologist, 18, 385-394.

Hsieh, S.L.J., \& Tori, C.D. (2007). Normative data on crosscultural neuropsychological tests obtained from Mandarinspeaking adults across the life span. Archives of Clinical Neuropsychology, 22, 283-296.

Johnson, D.K., Storandt, M., \& Balota, D.A. (2003). Discourse analysis of logical memory recall in normal aging and in dementia of the Alzheimer type. Neuropsychology, 17, 82-92.

Lamar, M., \& Resnick, S.M. (2004). Aging and prefrontal functions: dissociating orbitofrontal and dorsolateral abilities. Neurobiology of Aging, 25, 553-558.

Lecours, A.R., Mehler, J., Parente, M.A., Caldeira, A., Cary, L., Castro, M.J., Dehaut, F., Delgado, R., Gurd, J., de Fraga Karmann, D., Jakubovitz, R., Osorio, Z., Cabral, L.S., \& Junqueira, A.M.S. (1987). Illiteracy and brain damage: 1 . Aphasia testing in culturally contrasted populations (control subjects). Neuropsychologia, 25, 231-245.

Lin, C.H., Chiu, Y.C., Lee, P.L., \& Hsieh, J.C. (2007). Is deck B a disadvantageous deck in the Iowa Gambling Task? Behavioral and Brain Functions, 3, ArtID 16.

MacPherson, S.E., Phillips, L.H., \& Sala, D.S. (2002). Age, executive function and social decision making: a dorsolateral prefrontal theory of cognitive aging. Psychology and Aging, 17, 598-609.

Manly, J.J. (2008). Critical issues in cultural neuropsychology: profit from diversity. Neuropsychology Review, 18, 179-183.

Manly, J.J., \& Echemendia, R.J. (2007). Race-specific norms: using the model of hypertension to understand issues of race, culture, and education in neuropsychology. Archives of Clinical Neuropsychology, 22, 319-325.

Manly, J.J., Jacobs, D.M., Sano, M., Bell, K., Merchant, C.A., Small, S.A., \& Stern, Y. (1998). African American acculturation and neuropsychological test performance among nondemented 
community elders. Journal of the International Neuropsychology Society, 4, 77.

Manly, J.J., Jacobs, D.M., Touradji, P., Small, S.A., \& Stern, Y. (2002) Reading level attenuates differences in neuropsychological test performance between African American and white elders. Journal of the International Neuropsychology Society, 8, 341-348.

Nascimento, E. (2000). Adaptação e validação do teste WAIS-III para um contexto brasileiro. Tese de Doutorado não-publicada, Curso de Pós-graduação em Psicologia, Universidade de Brasília. Brasília, DF.

Ostrosky-Solis, F., Gutierrez, A.L., Flores, M.R., \& Ardila, A. (2007). Same or different? Semantic verbal fluency across Spanish-speakers from different countries. Archives of Clinical Neuropsychology, 22, 367-377.

Plumet, J., Gil, R., \& Gaonac'h, D. (2005). Neuropsychological assessment of executive functions in women: effects of age and education. Neuropsychology, 19, 566-577.

Radanovic, M., Mansur, L.L., \& Scaff, M. (2004). Normative data for the Brazilian population in the Boston Diagnostic Aphasia Examination: influence of schooling. Brazilian Journal of Medical and Biological Research, 37, 1731-1738.

Razani, J., Burciaga, J., Madore, M., \& Wong, J. (2007). Effects of acculturation on tests of attention and information processing in an ethnically diverse group. Archives of Clinical Neuropsychology, $22,333-341$

Reavis, R., \& Overman, W.H. (2001). Adult sex differences on a decision-making task previously shown to depend on the orbital prefrontal cortex. Behavioral Neuroscience, 115, 196-206.

Rosselli, M., Tappen, R., Williams, C., \& Salvatierra, J. (2006). The relation of education and gender on the attention items of the MiniMental State Examination in Spanish speaking Hispanic elders.
Archives of Clinical Neuropsychology, 21, 677-686.

Schmand, B., Smit, J.H., Geerlings, M.I., \& Lindeboom, J. (1997). The effects of intelligence and education on the development of dementia: a test of the brain reserve hypothesis. Psychological Medicine, 27, 1337-1344.

Schneider, D.D.G., \& Parente, M.A.M.P. (2006). O desempenho de adultos jovens e idosos na Iowa Gambling Task: Um estudo sobre a tomada de decisão. Psicologia: Reflexão e Crítica, 19, 442-450.

Sheehan, D.V., Lecrubier, Y., Sheehan, K.H., Amorim, P., Janavs, J., Weiler, E., Hergueta, T., Baker, R., \& Dunbar, G.C. (1998). The Mini-International Neuropsychiatric Interview (M.I.N.I.): the development and validation of a structured diagnostic psychiatric interview for DSM-IV and ICD-10. Journal of Clinical Psychiatry, 59 (Suppl 20), 22-33.

Tranel, D., Bechara, A., \& Denburg, N.L. (2002). Asymmetric functional roles of right and left ventromedial prefrontal cortices in social conduct, decision-making, and emotional processing. Cortex, 38, 589-612.

Verdejo-García, A., Bechara, A., Recknor, E.C., \& Pérez-García, M. (2007). Negative emotion-driven impulsivity predicts substance dependence problems. Drug and Alcohol Dependence, 91, 213-219.

Wechsler, D. (1997). WAIS-III: Escala de Inteligência Wechsler para Adultos-Manual. Adaptação e Padronização de Uma Mostra Brasileira por Elizabeth do Nascimento. São Paulo: Casa do Psicólogo.

Wood, S., Busemeyer, J., Koling, A., Cox, C.R., \& Davis, H. (2005). Older adults as adaptive decision-makers: evidence from the Iowa Gambling Task. Psychology and Aging, 20, 220-225.

Yechiam, E., Busemeyer, J.R., Stout, J.C., \& Bechara, A. (2005). Using cognitive models to map relations between neuropsychological disorders and human decision-making deficits. Psychological Science, 16, 973-978. 